\title{
Time-dependent prediction degredation assessment of neural-networks-based TEC forecasting models
}

\author{
Th. D. Xenos ${ }^{1}$, S. S. Kouris ${ }^{1}$, and A. Casimiro ${ }^{2}$ \\ ${ }^{1}$ Department of Electrical and Comp. Eng., Univ. of Thessaloniki, Greece \\ ${ }^{2}$ Department of Electrical and Comp. Eng., Univ. do Algarve, Faro, Portugal
}

Received: 5 March 2003 - Revised: 31 July 2003 - Accepted: 1 September 2003

\begin{abstract}
An estimation of the difference in TEC prediction accuracy achieved when the prediction varies from $1 \mathrm{~h}$ to 7 days in advance is described using classical neural networks. Hourly-daily Faraday-rotation derived TEC measurements from Florence are used. It is shown that the prediction accuracy for the examined dataset, though degrading when time span increases, is always high. In fact, when a relative prediction error margin of $\pm 10 \%$ is considered, the population percentage included therein is almost always well above the 55\%. It is found that the results are highly dependent on season and the dataset wealth, whereas they highly depend on the $f o \mathrm{~F} 2$ - TEC variability difference and on hysteresislike effect between these two ionospheric characteristics.
\end{abstract}

\section{Introduction}

The prediction of ionospheric total electron content (TEC) is a complex problem. The greatest contribution to the TEC is from the ionospheric F-layer, which is a significantly variable ionized region of the atmosphere, where the electron concentration and distribution is governed by not only solar and geomagnetic influences, but also by neutral-wind effects. The use of neural networks to predict values of ionospheric peak electron density or $f o \mathrm{~F} 2$ is now well established (Xenos, 2002). However, the variability of TEC is not governed by exactly the same factors as $f o \mathrm{~F} 2$, since important contributors to the TEC are also the topside ionosphere and influences from the plasmasphere above the F2-region (Ciraolo and Spalla, 1997).

In this paper, forecasting assessment of TEC, $1 \mathrm{~h}, 1-$, 2and 7-days ahead, using neural networks is performed. TEC recorded at Florence has been divided into two groups, one for training the neural network and the other for testing the predictions. The two periods include the peaks of solar cy-

Correspondence to: Th. D. Xenos

(tdxenos@vergina.eng.auth.gr) cles 21 and 22. The accuracy of forecasting TEC using a neural-network approach is assessed.

\section{Data and analysis}

Faraday-rotation measurements from geostationary satellites, recorded at Florence (Spalla et al., 1987), have provided a valuable archive of TEC over a long period. Hourly-daily values of TEC from the time period 1975-1982 have been used to train the neural network. Hourly-daily TEC values from a separate time period (1989-1991) have been used for testing the prediction accuracy of the models. Four models were developed for each month; namely a model for onehour (1-h), one-day (1-d), two-days (2-d) and seven-days (7-d) ahead. No prediction was attempted for April, due to the fact that there were no measured values available for this month during the years 1989-1991. To compensate for the solar and geomagnetic activity effects on the ionosphere, the corresponding F2-layer critical frequencies $(f o \mathrm{~F} 2)$ measured at Rome, a vertical incidence ionospheric station close to Florence, were also used as inputs to the neural networks. The characteristics of the neural networks used are summarized in Table 1.

The normalized differences (DTEC) between the predicted and the measured values have been calculated as:

$D T E C=\left(T E C_{\text {pred }}-T E C_{o b s}\right) / T E C_{o b s}$

where:

$T E C_{\text {pred }}$ the predicted TEC values

$T E C_{o b s}$ the observed TEC values

The results have been classified in steps of $10 \%$ between $-40 \%$ and $40 \%$ and the distributions have been computed. Then, the results of all four models for each month were compared with respect to the accuracy degradation observed together with the population completeness. 
Table 1. Neutral Network characteristics

\begin{tabular}{ll}
\hline Neural Network type & Multilayer perceptron \\
Training procedure & Back propagation. Batching with adaptable learning rate. \\
Number of Layers & 1 input -2 hidden -1 output \\
Neuron activation functions & Linear for the input and for the output layer, tanh for the hidden ones.
\end{tabular}

Table 2. Prediction error distribution of 1-h, 1-d, 2-d and 7-d predicted TEC-values

\begin{tabular}{lcccccccccccccccccccccc}
\hline & \multicolumn{1}{c}{$-20 \%$} & \multicolumn{1}{c}{$-10 \%$} & \multicolumn{1}{c}{$0 \%$} & \multicolumn{4}{c}{$0 \%$} & \multicolumn{4}{c}{$10 \%$} & \multicolumn{1}{c}{$20 \%$} \\
\hline & $1 \mathrm{~h}$ & $1 \mathrm{~d}$ & $2 \mathrm{~d}$ & $7 \mathrm{~d}$ & $1 \mathrm{~h}$ & $1 \mathrm{~d}$ & $2 \mathrm{~d}$ & $7 \mathrm{~d}$ & $1 \mathrm{~h}$ & $1 \mathrm{~d}$ & $2 \mathrm{~d}$ & $7 \mathrm{~d}$ & $1 \mathrm{~h}$ & $1 \mathrm{~d}$ & $2 \mathrm{~d}$ & $7 \mathrm{~d}$ & $1 \mathrm{~h}$ & $1 \mathrm{~d}$ & $2 \mathrm{~d}$ & $7 \mathrm{~d}$ \\
\hline Jan & 3.1 & 4.9 & 12.2 & 7.0 & 42.5 & 12.8 & 30.6 & 16.8 & 35.3 & 41.4 & 44.4 & 41.4 & 11.5 & 19.0 & 0.7 & 19.9 & 1.9 & 5.6 & 0.0 & 4.2 \\
Feb & 4.9 & 5.5 & 6.7 & 6.6 & 36.6 & 25.3 & 24.5 & 15.5 & 33.3 & 34.9 & 34.4 & 25.2 & 10.8 & 16.4 & 12.3 & 38.8 & 2.5 & 6.6 & 10.3 & 1.6 \\
Mar & 15.9 & 10.2 & 17.1 & 10.2 & 28.2 & 18.8 & 22.6 & 30.5 & 32.7 & 23.7 & 21.2 & 18.1 & 10.2 & 26.0 & 12.9 & 15.9 & 0.0 & 6.9 & 12.9 & 10.2 \\
May & 1.9 & 8.3 & 5.1 & 12.3 & 17.5 & 17.5 & 13.8 & 17.3 & 36.5 & 22.4 & 32.7 & 23.7 & 28.4 & 26.2 & 33.9 & 18.3 & 10.1 & 13.7 & 7.1 & 8.3 \\
Jun & 1.1 & 9.7 & 10.2 & 10.9 & 9.3 & 27.4 & 18.7 & 19.0 & 31.9 & 26.6 & 20.0 & 20.3 & 39.1 & 14.0 & 17.4 & 21.0 & 13.2 & 8.2 & 14.5 & 13.5 \\
Jul & 0.0 & 6.9 & 8.8 & 7.0 & 1.7 & 17.8 & 26.3 & 21.7 & 28.3 & 31.3 & 30.8 & 29.0 & 47.6 & 28.5 & 19.2 & 22.6 & 15.2 & 6.0 & 6.6 & 6.9 \\
Aug & 0.0 & 8.1 & 9.2 & 6.0 & 6.1 & 25.4 & 22.8 & 18.6 & 39.1 & 27.2 & 30.0 & 28.2 & 44.5 & 23.4 & 23.4 & 23.4 & 5.3 & 7.5 & 7.1 & 14.7 \\
Sep & 6.7 & 14.2 & 12.7 & 10.3 & 18.9 & 23.9 & 34.0 & 26.0 & 52.3 & 25.2 & 24.4 & 19.5 & 14.4 & 16.3 & 6.0 & 10.1 & 0.0 & 4.8 & 2.5 & 7.3 \\
Oct & 0.7 & 7.9 & 10.0 & 8.4 & 37.9 & 13.9 & 14.2 & 9.2 & 16.9 & 19.3 & 20.4 & 19.7 & 15.6 & 20.6 & 23.5 & 24.8 & 9.0 & 17.4 & 7.3 & 19.3 \\
Nov & 5.7 & 3.5 & 3.7 & 10.8 & 39.0 & 15.7 & 20.5 & 11.1 & 25.6 & 44.6 & 22.8 & 21.3 & 15.9 & 13.6 & 18.2 & 15.9 & 1.5 & 10.7 & 11.8 & 11.9 \\
Dec & 4.5 & 5.2 & 3.9 & 9.2 & 32.9 & 16.3 & 10.0 & 25.1 & 32.5 & 55.0 & 56.6 & 26.0 & 21.6 & 5.4 & 14.5 & 16.8 & 1.6 & 1.8 & 4.1 & 11.2 \\
\hline
\end{tabular}

\section{Results and discussion}

The results obtained are summarized in Table 2. In this table, the prediction error distribution of 1-h, 1-d, 2-d and 7-d in advance predicted TEC-values is presented in steps of $10 \%$ for the relative accuracy margin of $\pm 20 \%$. A positive value indicates that the TEC has been underpredicted by the neural network. It can be seen that the accuracy of the models though degrading as the prediction time span increases, is very satisfactory in the $\pm 10 \%$ accuracy margin since it is always above $55 \%$ and in several cases it reaches $89.5 \%$. The maximum prediction accuracy for the 1-h model was obtained for January and August. This is not the general case though, since maximum prediction accuracy highly depends on the TEC$f_{o} \mathrm{~F} 2$ variability difference (Xenos, 2002) and given that $f_{o} \mathrm{~F} 2$ is a key factor in the neural network prediction model, the above result is evident. The worst case for all models is found to be in March and September or October. This can be partly attributed to the fact that the available datasets for these months were very poor. This result, though reasonable considering the operation of the neural network method, certainly merits further investigation. It is also possible that the cause of this variation in the prediction error is linked to the approach of training the neural network on TEC from one solar cycle and predicting on a consecutive one (Feichter and Leitinger, 1997). On the other hand, this could be due to the fact that both TEC and $f o \mathrm{~F} 2$ exhibit stronger variability during the equinoxes (Kouris et al., 1999). It is also known that the $f_{o} \mathrm{~F} 2$ and TEC variabilities differ, to some extent at
Table 3. Prediction accuracy $(\mathrm{p} \%)$ and database completeness

\begin{tabular}{lccccc}
\hline & & $1-\mathrm{h}$ & $1-\mathrm{d}$ & $2-\mathrm{d}$ & 7-d \\
\hline \multirow{2}{*}{ Winter } & p\% & 91.8 & 85.4 & 88.4 & 88.4 \\
& Population \% & 40.4 & 38.6 & 37.1 & 30.5 \\
\multirow{2}{*}{ Spring } & p\% & 90.7 & 86.9 & 89.7 & 82.4 \\
& Population \% & 30.3 & 30.3 & 29.9 & 29.9 \\
\multirow{2}{*}{ Summer } & p\% & 94.1 & 89.3 & 88.3 & 88.2 \\
& Population \% & 78.0 & 75.6 & 73.2 & 60.5 \\
Autumn & p\% & 86.7 & 83.9 & 77.3 & 75.2 \\
& Population \% & 84.8 & 82.4 & 79.8 & 65.8 \\
\hline
\end{tabular}

least due to the fact that the topside ionosphere and influences from the plasmasphere above the F2-region are important contributors to the TEC (Ciraolo and Spalla, 1997). If this is taken into account, then the error increase seems reasonable. Table 3 shows the overall predicted TEC population percentage lying within the $\pm 20 \%$ margin with respect to the database completeness. It can be seen here that generally the prediction accuracy depends more on the ionospheric variability that on the database completeness. Yet, this correlation, although evident, it is very difficult to be expressed by a closed form, since the TEC- $f \circ \mathrm{F} 2$ seasonal variability is not accounted for here.

Figure 1 presents the monthly $-10 \%,+10 \%$ prediction 


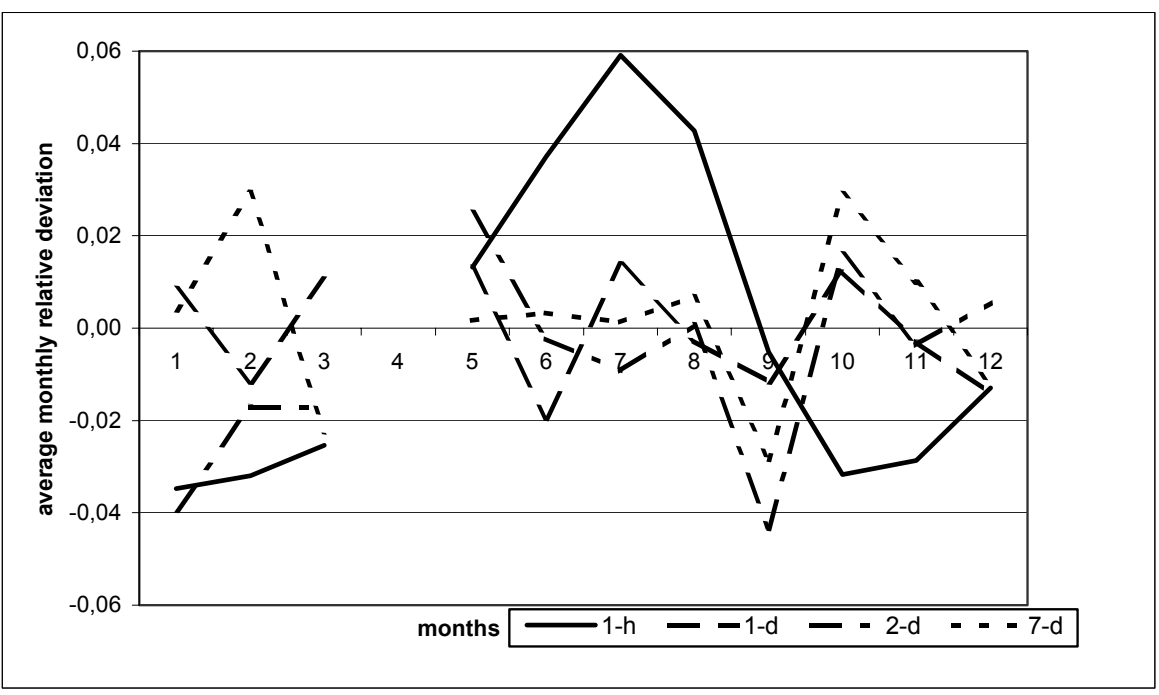

Fig. 1. Presentation of the monthly $-10 \%,+10 \%$ prediction error variation for each model. error variation. It can be seen that the 1-h before model, although more accurate (on the average) than the rest of the models, presents almost always the strongest variability. Moreover, from this figure it can also be observed that the average relative deviation becomes smallest for the 1-d model, whereas it is getting larger as the prediction margin increases, though it never reaches the accuracy of the 1-h model. This is very interesting, since it could give some evidence on the correlation distance of the TEC data. It seems that it is maximized when values with 24-h difference are considered. Of course this could be valid in all cases except for those of a disturbed ionosphere; yet since no discrimination between quiet and disturbed ionosphere is done in the present study, this assumption cannot be proved. On the other hand this could be due to the hysteresis like effect between $f o$ F2-TEC (Kouris and Fotiadis, 2002). In fact (Kouris et al., 2003) it was shown that there are two different values of TEC (or $f o \mathrm{~F} 2$ ) for a given value of $f o \mathrm{~F} 2$ (or TEC) and it is evident that for a given value of TEC the afternoon values of $f o \mathrm{~F} 2$ are higher than the morning ones and vice-versa. Of course, this is not easily interpreted and certainly deserves more investigation.

Acknowledgements. We herewith would like to thank Dr Paolo Spalla from IROE-Florence both for providing the TEC dataset and for helping us in understanding it in depth.

\section{References}

Ciraolo, L. and Spalla P.: Comparison of ionospheric total electron content from the navy Navigation Sattelite System and the GPS, Radio Science, 32.3, 1071-1080, 1997.

Feichter E. and Leitinger R.: A 22-year cycle in the F-layer ionisation of the ionosphere, Ann. Geophysicae, 15, 8, 1015-1027, 1997.

Kouris, S. S. and Fotiadis, D. N.: Ionospheric variability: A comparative statistical study, Adv. Space Res., 29(6), 977-985, 2002.

Kouris, S. S., Fotiadis, D. N., and Xenos, T. D.: On the day to day variation of $f o \mathrm{~F} 2$ and M(3000)F2, Adv. Space Res., 22.6, 873876, 1998.

Kouris, S. S., Fotiadis, D. N., and Zolesi, B.: Specifications of the Fregion variations for quiet and disturbed conditions, Phys. Chem. Earth, 24.4, 321-327, 1999.

Kouris, S. S., Xenos, T. D., Polymeris, K., and Stergiou D.: Some remarks on the relationship between TEC and $f o F 2$, 2nd Workshop COST 271 on Products for ITU-R and other radiocommunication applications, Faro Portugal, Oct., 2002.

Spalla, P., Capannini, E., and Ciraolo, L.: Sirio: A good Chance for Eight Years of Ionospheric Research, Alta Frequenza, LVI, 1-2, 167, 1987.

Xenos, Th. D.: Neural Network Based Single Station Models of the $f o \mathrm{~F} 2$ and $\mathrm{M}(3000) \mathrm{F} 2$ Ionospheric Characteristics, URSI 99, XXVI General Assembly, 1999.

Xenos, Th. D., Spalla, P., and Mitchell, C.: Neural Network based TEC forecasting models, paper 0940 presented at AP-2000 meeting at Davos, Switzerland, 2000.

Xenos, Th. D.: The effects of $f_{o} \mathrm{~F} 2$ variability on TEC prediction accuracy, Annals of Geophysics, 45.1, 191-200, 2002. 